\title{
Beteiligung und Streitkräfte - Ein demokratisches Prinzip setzt sich in europäischen Armeen durch
}

\author{
Andreas D. Prüfert*
}

\begin{abstract}
Co-determination and participation are normal usual principles in democracies. Until now this is not true for the military part of many societies. Even today there are military leaders who would prefer armed forces without any right of participation. This article comprises five chapters in which firstly the beginnings of co-determination in Germany are described. It is followed secondly by a historical review of participation in the German military before the situation of codetermination and participation in the Bundeswehr of today is analyzed. In a digression the normative requirements of German military leaders are discussed. The last chapter is about participation in other (European) armed forces. Finally the reader will find recent literature on the issue.
\end{abstract}

Keywords: Co-determination; Innere Führung; Bundeswehr; European Leadership Agency; Citizen in Uniform

\section{Einführung}

$\mathrm{M}$ itbestimmung innerhalb der militärischen Teilbereiche von Gesellschaften zählt vielfach eher zu den außergewöhnlichen Verhaltensmöglichkeiten. Auch in den deutschen Streitkräften gibt es teilweise immer noch Vorbehalte vor allem höherer Offiziere gegenüber zu intensiver Nutzung der Rechte des einzelnen Soldaten. Formal dulden sie zwar die Mitwirkungsrechte, aber insgeheim denken sie, wäre eine Armee nach amerikanischem, französischem oder englischem Muster wohl doch effektiver und daher wünschenswert. Nun gibt es nahezu seit Aufstellung der neuen deutschen Streitkräfte nach dem Zweiten Weltkrieg klare rechtliche Regelungen, die nicht einfach ignoriert werden können. Die Veränderungen nach Ende des OstWest-Konfliktes lassen allerdings Befürchtungen wachsen, dass in einer künftigen deutschen Freiwilligenarmee eher militärische Organisationsprinzipien auch dort wieder Platz greifen könnten, wo sie vom militärischen Auftrag nicht zwingend erforderlich wären. Auch die zunehmende Multinationalität lässt in deutschen Streitkräften die Frage auftauchen, ob die erworbenen Rechtspositionen deutscher Soldaten in Bezug auf Mitbestimmung auf dem Altar einer Gemeinsamen Außen- und Sicherheitspolitik mit dem kleinsten gemeinsamen Nenner geopfert - bzw. zumindest erheblich geschwächt - oder als entscheidende Merkmale von Streitkräften in einer Demokratie nach unserem Verständnis gegenüber anderen Nationen verteidigt und begründet werden.

In dem vorausgehenden Abschnitt sind bereits alle Aspekte angeschnitten, die bei der Behandlung des Themas angesprochen werden müssen. Zunächst ist eine isolierte Betrachtung lediglich des militärischen Bereiches nicht weiterführend. Da das Militär heute in Deutschland nicht mehr ein Staat im Staate ist, sondern überwiegend als ein Beruf wie jeder andere mit einigen Besonderheiten wahrgenommen wird, gibt es erhebliche Einflüsse aus der Gesellschaft auf das Militär. Die in der übrigen Gesellschaft vorhandenen

* Andreas D. Prüfert, Generalsekretär EUROMIL, Brüssel, Belgien.
Mitbestimmungsmöglichkeiten haben damit auch Auswirkungen auf den gesellschaftlichen Teilbereich Militär.

In Demokratien werden Einschränkungen von Rechten und die Gewährung besonderer Machtbefugnisse in der Regel begrenzt und ausgeglichen durch besondere Rechte und Schutzmöglichkeiten der Betroffenen. Von daher muss in jedem Fall die rechtliche Situation innerhalb der Bundeswehr in Bezug auf Mitbestimmung untersucht werden.

Deutsche Streitkräfte werden üblicherweise zusammen mit anderen, verbündeten Streitkräften eingesetzt, wenn es sich nicht um rein nationale Hilfeleistungen wie z. B. beim OderBruch handelt. Auch in Friedenszeiten sind die deutschen Großverbände inzwischen nahezu alle in multinationale Strukturen eingebunden. Obwohl die Disziplinargewalt und Personalführung bis heute durch deutsche Vorgesetzte ausgeübt wird, sind die Auswirkungen zunehmender Multinationalität auf die Mitbestimmungsrechte deutscher Soldaten nicht umfassend analysiert.

Die drei eben genannten Aspekte des Themas Mitwirkung und Mitbestimmung in europäischen Streitkräften haben jeweils einen unterschiedlichen Fokus. Zum einen den breiten Fokus der deutschen Gesellschaft, zum anderen den engeren der Teilgesellschaft Militär in Deutschland und schließlich einen über die deutsche Gesellschaft hinausgehenden. Letzterer bleibt allerdings in dieser Abhandlung unscharf, da er nur angerissen werden kann. Eine umfassende Behandlung des Themas Mitbestimmung in anderen Armeen und entsprechender Zusammenhänge mit der Bundeswehr ist weder vom Umfang noch von der Datenlage her möglich.

\section{Mitbestimmung in Deutschland}

Demokratie hat in Deutschland keine sehr lange Tradition. Es ist daher nicht verwunderlich, dass auch die institutionelle Verankerung von Mitbestimmung noch nicht sehr lange gegeben ist. Auch die Prägung deutscher Familienstrukturen durch patriarchalische Orientierungen ist bis weit 
über die Mitte des letzten Jahrhunderts vorherrschend gewesen und ließ schon innerhalb der Familien kaum Mitbestimmung zu.

Die Anfänge der allgemeinen Gewerkschaftsbewegung in Deutschland finden sich vor nun etwa 150 Jahren, als 1848 die ersten örtlichen Arbeiterverbände gegründet wurden. Allerdings gab es in den nächsten Jahrzehnten viele Aufund Abwärtsbewegungen im Ausbau der entsprechenden Rechte. Restriktive Phasen wie unter dem Sozialistengesetz oder im Dritten Reich, als im Jahre 1934 die freien Gewerkschaften abgeschafft wurden, verhinderten eine breite Durchsetzung der Mitbestimmungsmöglichkeiten der Arbeitnehmer.

Als sich nach dem Zweiten Weltkrieg die Bundesrepublik Deutschland mit dem Grundgesetz eine neue, demokratische Ordnung gab, wurden nicht nur freie Gewerkschaften wieder zuge-lassen, sondern die Möglichkeit, sich in Gewerkschaften oder Berufsverbänden zur Wahrung der Interessen zusammenzuschließen, wurde zum Grundrecht erhoben. Wörtlich heißt es in Artikel 9 Grundgesetz (Vereinigungs-, Koalitionsfreiheit): "Das Recht, zur Wahrung und Förderung der Arbeits- und Wirtschaftsbedingungen Vereinigungen zu bilden, ist für jedermann und für alle Berufe gewährleistet.« (Grundgesetz 1999: 15). Innerhalb der Betriebe und des öffentlichen Dienstes wurde Mitbestimmung ebenfalls rechtlich festgeschrieben und zwar im Betriebsverfassungsgesetz (1952) und im Personalvertretungsgesetz (1955). Auch diese ersten gesetzlichen Grundlagen in der Nachkriegszeit waren durch die eher konservative Grundhaltung in der Gesellschaft geprägt. Veränderungen in der Arbeitswelt und der beginnende Wertewandel Ende der 60er Jahre schufen das Klima für einen Ausbau der Mitbestimmungsrechte (Vgl. Gronimus 1996: XV). Ein neues Betriebsverfassungsgesetz (1972) und ein Bundespersonalvertretungsgesetz (1974) waren die Folge dieses Wandels. Gerade in letzterem wurden die Mitbestimmungsrechte gegenüber den schwächeren Mitwirkungs- und Anhörungsrechten ausgebaut (Siehe Gronimus 1996: XV).

Trotz all dieser gesetzlichen Grundlagen und Erfahrungen belegt Deutschland hinsichtlich des Organisationsgrades in Gewerkschaften lediglich einen mittleren Platz. So waren etwa 1993/94 von den Beschäftigten in Frankreich gerade einmal $10 \%$ gewerkschaftlich organisiert, in Deutschland $36 \%$ und in Schweden 82\% (Erich Schmidt Verlag 1994). Um die tatsächliche Mitbestimmung beurteilen zu können, muss allerdings die Wahrnehmung der Rechte analysiert werden. Mit Hilfe dieser Zahlen und der Darstellung der rechtlichen Verankerung können lediglich die Möglichkeit und das Ausmaß der Mitbestimmung festgestellt werden.

$\mathrm{Ob}$ jemand seine gegebenen Rechte auch tatsächlich nutzt, hängt wie bei anderen Einstellungen auch unter anderem von der Verankerung im persönlichen Wertesystem ab. Änderungen der Wertsysteme oder einiger Werte nicht nur bei einzelnen, sondern bei einer Mehrzahl von Bürgern einer Gesellschaft (Wertewandel) können daher auch Auswirkungen auf Mitbestimmungsfragen haben. Der Wandel vom ,traditionellen' zum ,posttraditionellen' Paradigma ist auch charakterisiert durch eine Verabschiedung von der ,Ruhe und Ordnung'-Einstellung hin zu einer Betonung eher individueller Werte. In unserem Zusammenhang wird als charakterisierender Aspekt für eine posttraditionelle Orientierung u.a. die »Betonung von Beteiligungs- und Mitspracherechten im Beruf und bei politischen Entscheidungen « genannt (Kohr 1996: 16). Trifft: dies zu, so wird der Wandel auch entsprechende Auswirkungen bei der Rekrutierung für das Teilsystem Militär mit seinen Einschränkungen der Beteiligungsrechte und Mitsprachemöglichkeiten haben.

In seinen Auswirkungen noch nicht breit analysiert sind die gesellschaftlichen Folgen der Entwicklung von der Industriezur Informationsgesellschaft und z.B. der Globalisierung der Wirtschaft. Interessant wäre z. B. die Untersuchung, warum beim Zusammenschluß von Daimler und Chrysler das deutsche Betriebsverfassungsmodell übernommen wurde und welche Erfahrungen der neue Konzern und die Mitarbeiter damit gemacht haben.

\section{Beteiligung im deutschen Militär - ein historischer Rückblick}

Die Anfänge der Mitbestimmung und Interessenvertretung im deutschen Militär liegen nicht ganz so weit zurück wie in der Gesellschaft. Dennoch wundert man sich vielleicht, dass die Institution des Vertrauensmannes bereits erstmals im Befehl Nr. 1 des Generalmajors Maerker vom 14.12.1918 erwähnt wird. Allerdings hat die Anordnung der Wahl von Vertrauensmännern in dem Freiwilligen Landjägerkorps noch nichts mit der Schaffung demokratischer Traditionen zu tun, sondern eher mit der zusätzlichen Möglichkeit, eine Abwahl des Vorgesetzten zu verhindern (Vgl. Gronimus 1994: 1).

Mit der Bildung der ,vorläufigen' Reichswehr wurde 1919 auch die Einrichtung des Vertrauensmannes eingeführt. Daneben wurden mit Verordnung 1920 eine sogenannte ,vorläufige Heereskammer' und ,vorläufige Marinekammer' als konsultierende Institutionen geschaffen. "Hierbei war vor allem an eine Mitwirkung bei der Beratung von Gesetzesvorlagen gedacht, die sich auf die Streitkräfte bezogen, sowie an eine Begutachtung von Wünschen und Anregungen grundsätzlicher Art aus diesen Bereichen.« (Rohde 1991: 11) Im Wehrgesetz von 1921 wurden alle drei Institutionen endgültig bestätigt. Dennoch verwehrte das Gesetz im Widerspruch zur Reichsverfassung den Soldaten das Koalitionsrecht weitgehend (Vgl. Giesen 1995: 12).

Obwohl die Wirksamkeit dieser Einrichtungen wohl eher gering war, zerschlugen die neuen Machthaber rasch wieder diese ersten Ansätze. Mit dem Zweiten Gesetz zur Änderung des Wehrgesetzes vom 20. Juli 1933 wurden alle drei Einrichtungen abgeschafft. Offensichtlich passten sie nicht zum nun propagierten ,Führerprinzip'.

Erst 1950 finden sich wieder Überlegungen zur Mitbestimmung in künftigen deutschen Streitkräften. In der sogenannten ,Himmeroder Denkschrift' wurden die Grundzüge eines westdeutschen Verteidigungsbeitrages beschrieben. 
Auch über das ,Innere Gefüge' dieser Streitkräfte hatten sich Experten in einer Klausurtagung in dem Eifel-Kloster Himmerod Gedanken gemacht. Man wollte zwar grundlegend Neues schaffen, konnte sich aber auch nicht von traditionellen Vorstellungen lösen. So wurde u.a. vorgeschlagen, dass die Mitgliedschaft in Gewerkschaften während der Zugehörigkeit zu den Streitkräften ruhen sollte (Rautenberg/Wiggershaus 1977: 54).

Obwohl schließlich durch die Wehrgesetze viele Rechte für Soldaten eingeschränkt wurden, hat der Gesetzgeber ihnen 1956 das Koalitionsrecht voll gewährt. Auch die Wahl von Vertrauensmännern war im Soldatengesetz vorgeschrieben. Soldaten in Dienststellen und Einrichtungen ohne Vertrauensmänner wählen Vertretungen nach dem Personalvertretungsgesetz. Eine Anpassung ohne grundlegende Neuerung erfuhren das Soldatengesetz und das Vertrauensmännerwahlgesetz im Jahre 1975. Die Rechte der Vertrauensmänner wurden zwar 1982 mit dem Inkrafttreten der Zentralen Dienstvorschrift ZDv 10/2 weiter gestärkt, aber eine deutliche Verbesserung der Mitbestimmungsrechte brachte erst das Beteiligungsgesetz vom 22. Januar 1991. Das ,Soldatenbeteiligungsgesetz' findet sich als Artikel 1 in diesem Beteiligungsgesetz. Wegen vieler Ungereimtheiten und strittiger Fragen wurde das Gesetz bereits 1997 mit einem Ersten Änderungsgesetz neu gefasst (Vgl. DBwV 1997: I-VIII).

Wohl eher als kurzfristige Episode ohne nachhaltige Wirkungen ist die Entwicklung zahlreicher Mitbestimmungsmodelle Anfang der siebziger Jahre zu sehen. Dies ging von Überlegungen zu einem Dienstplanausschuß (Böblinger Modell), über ein Soldaten-Mitarbeiter-Gremium (SoMiG-
Modell), einen Vorschlag mit der Bildung von Bundeswehrräten (Darmstädter Modell) bis zur Ablösung der Vertrauensmänner durch Soldatenvertreter (Siegener Modell). Diese Vorschläge wurden auch in Fachzeitschriften diskutiert und fanden zum Teil Niederschlag in den Berichten des Wehrbeauftragten, führten in der Praxis aber nicht zu wesentlichen Verbesserungen der Beteiligung von Soldaten (Vgl. Heckner 1986: 570f.).

\section{Interessenvertretung und Mitbestimmung in der Bundeswehr heute}

Nach diesem notwendiger Weise kurzen Rückblick auf die Entwicklungsgeschichte der Mitbestimmungs- und Koalitionsrechte in den Streitkräften sollen nun die Rechte in der Bundeswehr von heute systematisch dargestellt werden. Dabei orientiere ich mich an einer Gliederung in einem Vortrag des zuständigen Referatsleiters im Führungsstab der Streitkräfte, Oberst i.G. Schwarz, bei einer Tagung des Arbeitskreises Militär und Sozialwissenschaften zum Thema ,Mitbestimmung in den Streitkräften' (Schwarz 1991: 57f.). Schwarz unterscheidet zunächst zwischen direkter und indirekter Beteiligung. Dabei ist unter direkter Beteiligung die »Beteiligung des einzelnen Individuums « und unter indirekter die »Beteiligung durch Einschaltung gewählter Personen« (Schwarz 1991: 53) zu verstehen.

In der folgenden Übersicht sind die aktuellen Möglichkeiten der Beteiligung, wie sie Schwarz aufgelistet hat (Schwarz 1991: 57f, ergänzt durch den Autor), zusammengestellt:

Tabelle 1: Beteiligungsformen in der Bundeswehr

\section{Direkte oder unmittelbare Beteiligung}

Führungsmethoden wie unter anderem

- Delegation von Handlungsverantwortung

- Führung mit Stäben

- Kooperativer Führungsstil

Rechtlich verankerte Formen

- Beschwerderecht mit seinen Rechtsbehelfen

- Antrags-, Anhörungs- und Vorspracherecht

- Begrenzung der Gehorsamspflicht mit der Pflicht zur Meldung

- Beurteilungsverfahren mit Anhörung und Gegenvorstellung

- Mitarbeit in Ausschüssen (v. a. Betreuungs-, Kantinen- und Küchenausschuss)

- Teilnahme als ehrenamtlicher Richter am Truppendienstgericht

Individuell praktizierte Formen

- Erfahrungs- und Zustandsberichte

- Lehrgangskritik

- Verbesserungsvorschläge

- Private Veröffentlichungen mit dienstlichem Bezug

\section{Indirekte Beteiligung}

- $\quad$ Einschaltung der Vertrauensperson oder entsprechender Gremien nach dem Soldatenbeteiligungsgesetz

- Einschaltung der Personalvertretung nach dem Bundespersonalvertretungsgesetz

- Einschaltung eines Vermittlers nach der Wehrbeschwerdeordnung

- Einschaltung eines Vertreters in einem Ausschuss

- Einschaltung eines Vertreters des Deutschen BundeswehrVerbandes 
Als Vertreter des Ministeriums erklärt Schwarz »Wir wollen, daß in der Truppe ein Betriebsklima entsteht, in dem Beteiligung in allen den einzelnen Soldaten betreffenden Alltagsdingen einen Grad der Normalität erreicht, dem sich niemand entziehen kann « (Schwarz 1991: 58). Zum damaligen Zeitpunkt räumt er Mängel vor allem bei den Mannschaften im Hinblick auf Einwirkungs- und Mitgestaltungsmöglichkeiten ein. Hier fehle »ein Gesamtkonzept « (Schwarz 1991: 58).

Diese Erkenntnisse finden sich in noch deutlicherer Form in einer Studie zur, Verbesserung der Mitwirkung von Mannschaften' (AStudÜbBw 1990). Nach recht kritischen Ergebnissen aus der sogenannten Erziehungsstudie hatte der Bundesminister der Verteidigung entschieden, die »Mitwirkung von Grundwehrdienstleistenden im Sinne des Führens mit Auftrag bei der Ausgestaltung des Dienstalltags zu stärken « (AStudUbBw 1990: 11). Dabei sollten Schwerpunkte der Studie zum einen »die Analyse und Bewertung der normativen Vorgaben der Streitkräfte«, zum anderen das »Aufzeigen von praktischen Vorschlägen« (AStudUbBw 1990: 11) sein.

Die Studie kommt bei der Auswertung von Erfahrungen der Truppe zu einem niederschmetternden Ergebnis: „Die Diskrepanz zwischen dem Anspruch der Führung, den mündigen Staatsbürger in Uniform zum mitdenkenden Gehorsam $\mathrm{zu}$ führen, auszubilden und $\mathrm{zu}$ erziehen und der in weiten Bereichen der Streitkräfte täglich erlebten Realität der Bevormundung und Unselbständigkeit der Mannschaften ist eklatant, Gegenstand von Unmut und Resignation in der Truppe, Grund für das Abtauchen von Wehrdienstleistenden und in hohem Maße mitverantwortlich für mangelnde Motivation « (AStudÜbBw 1990: 144). Auch in der Auswertung einer Umfrage des Sozialwissenschaftlichen Instituts der Bundeswehr finden sich recht hohe Zustimmungswerte zur Wichtigkeit der Mitbestimmung für Soldaten. Von 2.490 befragten Soldaten halten 73,7\% Mitbestimmung für Soldaten für eher wichtig oder sehr wichtig (SOWI 1996: 72).

Die normativen Vorgaben in Vorschriften, Erlassen, Aussagen höherer Führer sehen eine umfassende Beteiligung vor, die tatsächliche Umsetzung im Truppenalltag ist aber äußerst mangelhaft. Um diesem Befund abzuhelfen, werden in der Studie insgesamt 53 Maßnahmen zur Verbesserung vorgeschlagen. Aus der folgenden Übersicht ist erkennbar, in welchen Bereichen die Beteiligungsmöglichkeiten verbessert werden sollen:

Tabelle 2: Bereiche des Maßnahmenkatalogs

1 Maßnahmen bei Dienstantritt

2 Mitwirkung beim täglichen Dienst

2.1 Dienstbesprechungen und Informationsgespräche

2.2 Innendienst

2.3 Ausbildung

2.4 Betreuung und Fürsorge

2.5 Besondere Maßnahmen

3 Mitwirkung bei Personalmaßnahmen

$4 \quad$ Mitwirkung vor der Entlassung

5 Teilstreitkraftgebundene Maßnahmen und Vorschläge
Einen Ausfluss in Form einer Weisung zur Umsetzung der Vorschläge oder zumindest eine breite Diskussion der Studie hat es nicht gegeben. Vielmehr wurde die Studie als Verschlusssache eingestuft. Erst nach fast acht Jahren (1997) wurde diese Einstufung aufgehoben. Offensichtlich bestehen bis heute bei vielen Vorgesetzten Unsicherheiten in der Abgabe von Macht und Abneigung gegenüber Mitwirkungsmöglichkeiten und deren Wahrnehmung durch die Untergebenen. Insgesamt scheint aber das gesamte militärische Sozialisationssystem die Ausprägung entsprechender Qualifikationen bzw. Fähigkeiten der Vorgesetzten nicht gerade zu fördern. In Seminaren zum Thema »Beteiligungsrechte«, welche die Karl-Theodor-Molinari-Stiftung, das Bildungswerk des Deutschen BundeswehrVerbandes, anbietet, finden sich immer häufiger auch Disziplinarvorgesetzte.

Eine wesentliche Veränderung - auch im Bereich der Beteiligung - bringt die Neuorientierung der Bundeswehr hin zu einer Einsatzarmee. Die Zusammensetzung der Einsatzkontingente aus vielen Einheiten schränkt ohne böse Absicht dahinter die Mitwirkungsmöglichkeiten ein. Auch das Klima innerhalb der Armee hat sich gewandelt, nachdem im Ausland vor allem Zeit- und Berufssoldaten eingesetzt sind, abgesehen von einigen sogenannten FWDL (freiwillig längerdienende Wehrpflichtige), die aber eigentlich als Zeitsoldaten anzusehen sind. Die Berichte des Wehrbeauftragten zeigen diese Klimaveränderung deutlich auf. Dabei ist ein Trend zu erkennen, dass Befehl und Gehorsam auch in solchen Bereichen wieder Platz greift, in denen es eher um Beratung mit Untergebenen und deren Beteiligung geht.

\section{Exkurs: Normative Vorgaben für die Mitwirkung in den deutschen Streitkräften}

Eine Demokratie ohne Engagement der Bürger ist für uns nicht vorstellbar, im Gegenteil ist und bleibt ein demokratisches Staatswesen erst lebendig, wenn sich seine Bürger an Entscheidungen beteiligen. Dies kann auf unterschiedlichste Weise erfolgen, z.B. durch Beteiligung an Wahlen auf den unterschiedlichsten Ebenen, durch Beteiligung in Schulen, Vereinen, Bürgerinitiativen, Nachbarschaftshilfen oder anderen ehrenamtlichen Aufgaben. Natürlich auch durch Beteiligung am eigenen Arbeitsplatz.

Mitwirkung an unserem persönlichen Lebensumfeld ist für uns ein notwendiges und selbstverständliches demokratisches Prinzip, um dem einzelnen unter Berücksichtigung der Mitmenschen soviel Selbstentfaltungsmöglichkeiten wie möglich zu bieten und seine Menschenwürde $\mathrm{zu}$ achten. »Dabei kommt es nicht so sehr darauf an, inwieweit der einzelne von seinen Beteiligungsrechten Gebrauch macht, vielmehr ist es wichtig, dass die Beteiligung als demokratisches Prinzip überhaupt besteht und von jedem Menschen angewandt werden kann, vor allem dort, wo er tatsächlich betroffen ist (AStudÜbBw 1990: 20).

Bei Aufstellung neuer Streitkräfte nach dem Zweiten Weltkrieg wurde das Leitbild des Staatsbürgers in Uniform entwickelt. Man ging davon aus, dass der Soldat grundsätzlich die 
gleichen Rechte und Pflichten wie jeder andere Bürger hat. Seine Rechte dürfen nur im Rahmen der Erfordernisse des militärischen Dienstes eingeschränkt werden. Das bedeutet auch, dass der Soldat in Bezug auf Mitwirkungsrechte grundsätzlich die gleichen Rechte wie jeder andere Bürger haben muss. Rechtlich umgesetzt ist diese Forderung durch die Bestimmungen des Soldatenbeteiligungsgesetzes und des Bundespersonalvertretungsgesetzes. Es gibt allerdings bis heute unterschiedliche Auffassungen über die Reichweite dieser beiden Regelungen. Vor allem der Deutsche BundeswehrVerband fordert immer wieder die Einbeziehung aller Bereiche in das Bundespersonalvertretungsrecht (vgl. Gertz 1991: 64). Die militärische Führung will allerdings bis heute nicht so weit gehen.

Allgemein werden als Grenze der unmittelbaren Mitwirkung in Streitkräften bei uns folgende Prinzipien bzw. Bereiche gesehen:

Tabelle 3: Grenzen unmittelbarer Beteiligung in den Streitkräften

$>$ Das Prinzip von Befehl und Gehorsam

$>$ Die Einhaltung der Entscheidungs- und Führungsstrukturen

$>$ Das Prinzip der Unteilbarkeit von Führungs- und Entscheidungsverantwortung

Die weiter oben erwähnte Studie kommt zu dem Schluss, dass ein erhebliches Potenzial für die Erweiterung der Mitwirkungsmöglichkeiten besteht. "Die durch Wehrpf1ichtige, Zeitsoldaten und Reservisten in die Streitkräfte hineingetragenen Beteiligungserwartungen können in erheblichem Umfang durch entsprechendes Führungsverhalten erfüllt werden, ohne den gesetzlich festgelegten Auftrag zu berühren oder das Prinzip von Befehl und Gehorsam in Frage zu stellen.« (AStudÜbBw 1990: 268).

Das entsprechende Führungsverhalten, um Mitwirkung in größerem Umfang zu ermöglichen, scheint aber nicht genügend vorhanden. Nicht umsonst hat der Generalinspekteur festgestellt: »(...) ich sage in aller Deutlichkeit, dass Streitkräfte in einer modernen, demokratisch geprägten Gesellschaft allein nach dem Prinzip von Befehl und Gehorsam nicht mehr funktionsfähig sind. Vorgesetzte, die die Beteiligung der Soldaten als Last betrachten und ihr grundsätzlich ablehnend gegenüberstehen, entsprechen daher nicht den Anforderungen, die heute an unsere militärischen Führer gestellt werden müssen.« (Hausbeck 1999: 90).

Auch der letzte Bundesminister der Verteidigung stellte in seiner Rede bei seinem ersten Truppenbesuch fest, dass die neuen Aufgaben und die Veränderungen im Alltag der Bundeswehr erfordern, »die Grundsätze moderner Menschenfiihrung im Sinne von konkreter Mitgestaltung und Mitverantwortung $\mathrm{zu}$ überdenken und weiterzuentwickeln « (Scharping 1998: 2). Ähnlich sieht dies Keller, der die Anforderungen an den militärischen Führer nach dem Jahr 2000 analysiert hat. Er kommt in Bezug auf Menschenführung zu dem Schluss, dass zunehmend die Perspektive der Unterge- benen bei der Willensanordnung des Vorgesetzten mit einbezogen werden muss. "Damit wechselt sie die Perspektive gegenüber traditionellen Vorstellungen, die den Ausführenden vor dem Willen des Führers unberücksichtigt lässt, hin zu einer Sicht, die in der Anordnung bereits die Sicht des Ausführenden einbezieht. Obwohl neu für militärisches Handeln ist diese Perspektive bereits in der Konzeption Innere Führung angelegt und hat sich auch in den modernen Beteiligungsformen und Arbeitsstrukturen der Wirtschaft bewährt.«(Keller 1997: 9f.).

Von einem ganz anderen Ansatz her fordern ein junger Generalstabsoffizier und eine Wissenschaftlerin eine breite Beteiligung in den Streitkräften. Sie sehen in der völligen Neuorientierung der Bundeswehr, der "zweiten Neugeburt « (Hartmann/Strittmatter 1993b: 16), eine vergleichbar große Reformaufgabe wie bei der preußischen Heeresreform und der Neuaufstellung der Streitkräfte in den fünfziger Jahren. Als eine der drei Problemlösungsstrategien, die in den früheren Reformen angewandt wurden und auch heute notwendig seien, fordern sie die »kritische Mitwirkung und Mitbestimmung von Soldaten aller Dienstgradgruppen an der Lösung der Reformaufgaben « (Hartmann/Strittmatter 1993b: 17).

\section{Beteiligung in anderen (europäischen) Streitkräften}

Die ausgeprägten Mitbestimmungsrechte des Soldaten, wie sie in den deutschen Streitkräften verankert sind, können zu Recht als Ausnahme bezeichnet werden. Es gibt einige wenige Länder, in denen die Rechte ähnlich umfassend gewährleistet werden. Dies sind vor allem die skandinavischen, niederländischen und österreichischen Streitkräfte. Hier gehen die Rechte zum Teil sogar über die deutschen hinaus. In Schweden kann der Soldat zum Beispiel auf sein Streikrecht per Verfassung nicht verzichten. Allerdings wurde dieses Recht bisher noch nicht angewandt. In vielen anderen Nationen sind die Rechte aber sehr schwach ausgeprägt. So gibt es bis heute zum Beispiel in Spanien, Italien, Frankreich, Großbritannien oder den Vereinigten Staaten keine Möglichkeit für aktive Soldaten, entsprechend dem zivilen Koalitionsrecht einen Interessenverband oder eine Gewerkschaft für Soldaten zu gründen und sich darin zu artikulieren. Dies widerspricht den eigenen Verpflichtungen, welche diese Länder in der Organisation für Sicherheit und Zusammenarbeit in Europa (OSZE) eingegangen sind.

Der Forschungsstand auf diesem Gebiet ist bisher allerdings sehr begrenzt. Es gibt zur Zeit nur einige Arbeiten am Lehrstuhl von Professor Steinkamm an der Universität der Bundeswehr München. Dort sind rund 15 Dissertationen zum Thema »Europäischer Wehrrechtsvergleich « erstellt worden. Zur Zeit ist an den Lehrstuhl eine Studie zum Vergleich der unterschiedlichen Führungsphilosophien vergeben. Einen begrenzten Überblick - inzwischen allerdings veraltet - geben die Beiträge in dem Sammelband von Klein (1991). Hier finden sich zumindest Aussagen zu den Beteiligungsrechten der Soldaten in der Schweiz (Stadelmann 1991), Österreichs 
(Redl 1991) und Frankreichs (Hoffmann 1991; Bonnetête 1991). Einzigartig ist auch der Vergleich der zwei Vertretungsmodelle in der Deutsch-Französischen Brigade im gleichen Band (Robert 1991). Am Lehrstuhl von Professor Nolte wurde ein begrenzter Wehrrechtsvergleich zwischen neun Nationen im Rahmen einer Studie des Bundesministeriums der Verteidigung erstellt (Nolte 2002). Es wurden jeweils Experten von Universitäten gebeten, den Sachstand in ihrem Land darzustellen.

Auch andere Nationen beschäftigen sich gelegentlich mit solchen Vergleichen. Bekannt ist hierbei allerdings nur, dass in Italien eine solche Synopse mit den Systemen von wenigen ausgewählten Staaten vorgenommen wurde, weil man über die Einführung von Vertretungsrechten nachdachte (vgl. De Martin-Topranin 1995). Nach ersten umfangreicheren Untersuchungen über die Mitwirkung von Soldaten in den Streitkräften in den 80er Jahren (Caforio/Nuciari 1990) ist heute die Zeit gekommen, erneute Untersuchungen durchzuführen (vgl. Olivetta 2004). In Südafrika wurde innerhalb eines Prozesses vor dem Verfassungsgericht der Vergleich unter anderem mit dem Deutschen BundeswehrVerband herangezogen, um die Möglichkeit der Einführung von Gewerkschaften von Soldaten zu erstreiten (vgl. Klauer 1999). Auch in der Russischen Föderation ist man sehr an den Erfahrungen anderer Nationen interessiert, weil dort versucht wird, die Arbeit von Gewerkschaften im Militär gesetzlich zu regeln.

Viele Länder, die ihren aktiven Soldaten das Koalitionsrecht verweigern, begründen dies gar nicht oder mit pauschalen Hinweisen, dass dies die Disziplin oder die hierarchische Ordnung gefährden würde. In einer neueren Studie aus England (Akiwumi 2002) wird klar herausgearbeitet, dass es eigentlich keinen Grund gibt, den Soldaten das Koalitionsrecht $\mathrm{zu}$ verweigern. $\mathrm{Zu}$ den gleichen Ergebnissen gelangt ein ehemaliger Offizier, der seit Jahren versucht, für dieses Thema Aufmerksamkeit zu erregen, aber bisher kaum Reaktion von offizieller Seite erfährt. In einer Synopse zeigt er auf, dass mehr und mehr sich auch inoffizielle Vereinigungen gründen und die Stimmung der Soldaten sich langsam wandelt. Insgesamt kommt er zu dem Ergebnis, dass inzwischen alle Faktoren gegeben sind, um eine Interessenvertretung zu gründen (Bartle 2004).

Geht man davon aus, dass in Kürze das European Rapid Reaction Corps (ERRC) und mehrere »Battle Groups « sowie die NATO Response Force (NRF) einsatzbereit sind, aber bis heute wenig über die Folgen solcher politischen Entscheidungen für die Rechtsstellung der Soldaten nachge-dacht wurde, kann man sich nur wundem. Unsere Rückkehr zu einer sogenannten ,Normalität' im Reigen der anderen Nationen kann sehr rasch zu einem faktisch erheblichen Verlust an Mitbe-stimmungsrechten führen, selbst wenn formal die deutschen Soldaten einem deutschen Disziplinarvorgesetzten zugeordnet bleiben. In der Praxis entwickelte Modelle der Mitwirkung bei bi- oder multinationalen Großverbänden sind bisher kaum wissenschaftlich untersucht. Dabei gibt es zum Beispiel inzwischen beim DeutschNiederländischen Korps in Münster bi- und multinationale Consultation Committees, die zwar wenig formalisierte
Rechte haben, aber dennoch eine gewisse Mitwirkung zulassen.

Es ist daher längst überfällig, dass zum einen eine umfassendere Übersicht über die rechtlichen Bestimmungen in den Streitkräften zusammengestellt und diese übersetzt wird. Zum anderen muss schnellstmöglich ein Dialog auf hoher Ebene über Mitwirkungsmöglichkeiten bei multinationalen Verbänden begonnen werden. Hierzu muss man sich allerdings vorab auf nationaler Ebene verständigen, welche Rechte für uns nach dem Bild des Staatsbürgers in Uniform unabdingbar und damit nicht verhandelbar sind und welche gegebenenfalls zur Disposition gestellt werden könnten. Die Europäische Organisation der Militärverbände (EUROMIL) bemüht sich um einen solchen Dialog sowohl bei der NATO als auch bei den entsprechenden Gremien der Europäischen Union. So wurde kürzlich vorgeschlagen, in Ergänzung zu der in der Europäischen Verfassung vorgesehenen European Defence Agency eine European Leadership Agency einzurichten, welche die Transformation der nationalen Führungskonzeptionen koordiniert, minimale Standards für die Arbeitsbedingungen aller Soldaten entwickelt und die menschlichen und sozialen Rechte in den Curricula der Führerausbildung verankert.

\section{Literaturverzeichnis}

Akiwumi, Helena (o.J.) (2002, der Autor), Would an Armed Forces Federation undermine the Chain of Command? Study, (Manuscript), (o.O.).

Amt für Studien und Übungen der Bundeswehr (zitiert als: AStudÜbBw) (Hg.) (1990), Verbesserung der Mitwirkung von Mannschaften (Autor: Bernd Weber), Bergisch Gladbach (Studienteil und Anlagenband)

Bald, Detlef/Prüfert, Andreas (Hg.) (2002), Innere Führung. BadenBaden.

Bartle, Richard (2004), Independent Representation in the British Army: Has the Time Finally Arrived?, (Manuscript), Shrivenham.

Bonnetête, Jacques (1991), Der 'Conseil Superieur de la Fonction Militaire', in: Klein 1991: 129-140.

Bundeszentrale für politische Bildung (zitiert als: Grundgesetz) (1999), Grundgesetz für die Bundesrepublik Deutschland, Bonn.

Caforio, Giuseppe/Nuciari, Marina (1990), La rappresentanza militare in italia. Rivista Militare. Centro Militare di Studi Strategici, Rom.

De Martin-Topranin, Giancandido (1995), Ipotesi die Riforma della Rappresentanza Militare alle Luce della Evoluzione della Societa Nazionale e delle Nuove Esigenze delle Forze Armate, Rom.

Deutscher Bundeswehr-Verband (zitiert als: DBwV) (1997), Das neue Soldatenbeteiligungsgesetz- SBG - 1997. Die Bundeswehr, 45: 2, I-VIII.

Erich Schmidt Verlag (1994), Die Rolle der Gewerkschaften. Zahlenbilder 631400 .

EUROMIL (2004), Press Release (2004) 06. EUROMIL demands the establishment of a European Leadership Agency. Brussels, 22 June 2004.

Gertz, Bemhard (1991), Mitbestimmung in Streitkräften aus der Sicht der Soldatenvertretung, in: Klein 1991: 63-69.

Giesen, Hermann (1995), Deutscher Bundeswehr-Verband. Spitzenorganisation der Soldaten, Bonn.

Gronimus, Andreas (1994), Die Beteiligungsrechte der Vertrauenspersonen in der Bundeswehr. Erläuterungen zum Soldatenbeteiligungsgesetz. 2., überarb. und erw. Auflage, Berlin-BonnRegensburg.

Gronimus, Andreas (1996), Die Beteiligungsrechte der Personalvertretungen. Erläuterungen zum Bundespersonalvertretungsgesetz unter besonderer Berücksichtigung der Bundeswehr. 2., neubearb. und erw. Auflage. Regensburg-Bonn. 
Hartmann, Uwe/Strittmatter, Meike (1993a), Einleitung, in: Hartmann/Strittmatter 1993b: 15-20.

Hartmann, Uwe/Strittmatter, Meike (Hg.) (1993b), Reform und Beteiligung. Ideen und Konzepte für die Innere Führung in der Bundeswehr, Frankfurt.

Hausbeck, Volker (1999), Dynamischer Prozeß. Mitverantwortung, Mitwirkung und Beteiligung in den Streitkräften stärken das vertrauensvolle Miteinander von Vorgesetzten und Untergebenen. Truppenpraxis/Wehrausbildung, 43: 1, 89-92.

Heckner, Hans-Peter (1986), Mitwirkung und Mitbestimmung in Streitkräften, in: Kirchhoff 1986: 566-572.

Hoffmann, Gerard (1991), Die eingeschränkte Rechtsstellung der Soldaten in Frankreich - Mythos und Wirklichkeit, in: Klein 1991: 115-128.

Keller, Jörg (1997), Der militärische Führer nach dem Jahr 2000. Beiträge zu Lehre und Forschung 05/97, Hamburg.

Kirchhoff, Günter (Hg.) (1986), Handbuch zur Ökonomie der Verteidigungspolitik, Regensburg.

Klauer, Wolfgang P. (1999), Koalitionsfreiheit in Südafrika. Zulassung einer Gewerkschaft bei den Streitkräften durch das Verfassungsgericht, Deutsche Botschaft Pretoria, Bericht Nr. 894/1999, Pretoria.

Klein, Paul (Hrsg.) (1991), Mitbestimmung in den Streitkräften, Baden-Baden.

Kohr, Heinz-Ulrich (1996), Wertewandel und Soziomoral: Auswirkungen auf die Bundeswehr. SOWI-Arbeitspapier Nr. 95, Strausberg.

Nolte, Georg/Krieger, Heike (2002), Europäische Wehrrechtssysteme. Ein Vergleich der Rechtsordnungen Belgiens,

Dänemarks, Deutschlands, Frankreichs, Luxemburgs, der Niederlande, Polens, Spaniens und des Vereinigten Königreichs, Baden-Baden.

Olivetta, Eraldo (2004), Military Representation in the Italian Armed Forces in the Turn from Conscription to Professional Format, Manuscript, Turin.
Prüfert, Andreas (2000), Soldat und Mitbestimmung. In: Kümmel, Gerhard/Prüfert, Andreas D. (Hg.) (2000). Military Sociology. Baden-Baden: 407-417.

Prüfert, Andreas (2002), Innere Führung und das Leitbild vom »Europäischen Bürger in Uniform « - Über die Notwendigkeit einer bisher vermiedenen Debatte. In: Bald/Prüfert 2002: 79-90.

Prüfert, Andreas (2002a), Eine gemeinsame europäische Führungsphilosophie - Zusammenführen oder Fortbestehen nationaler militärischer Führungsgrundsätze? In: Hoyer, Werner/Kaldrack Gerd F. (Hg.) (2002). Europäische Sicherheits- und Verteidigungspolitik (ESVP). Baden-Baden.

Rautenberg, Hans-Jürgen/Wiggershaus, Norbert (1977), Die ,Himmeroder Denkschrift' vom Oktober 1950. Politische und militärische Überlegungen für einen Beitrag der Bundesrepublik Deutschland zur westeuropäischen Verteidigung, Karlsruhe.

Redl, Karl (1991), Mitbestimmungsmöglichkeiten im österreichischen Bundesheer, in: Klein 1991: 95-113.

Robert, Gilles (1991), Die Gegenüberstellung zweier nationaler Vertretungssysteme in der Deutsch-Französischen-Brigade, in: Klein 1991: 141-156.

Rohde, Horst (1991), Soldatische Beteiligungsrechte in der deutschen Militärgeschichte, in: Klein 1991: 9-18.

Scharping, Rudolf (1998), Zukunft der Bundeswehr - den Wandel gemeinsam gestalten. Ansprache des Bundesministers der Verteidigung, Rudolf Scharping, beim Zentrum Innere Führung in Koblenz am 9. November 1998, Redemanuskript, Berlin.

Schwarz, Günter (1991), Beteiligung in den Streitkräften aus der Sicht des Bundesministers der Verteidigung, in: Klein 1991: 5361.

Sozialwissenschaftliches Institut der Bundeswehr (zitiert als: SOWI) (1996), Bevölkerungsumfrage, Strausberg.

Stadelmann, Jörg (1991), Führungsbetreuung als Antwort auf die Herausforderung Mitbestimmung, in: Klein 1991: 83-94. 